\title{
ESTUDO IMAGINOLÓGICO DE UM CASO DE ANGINA DE LUDWIG
}

\section{IMAGING STUDY OF A CASE OF LUDWIG'S ANGINA}

\author{
Maria José Albuquerque Pereira de Sousa e Tucunduva* \\ Claudio Froes de Freitas* \\ Beatriz Ogassawara** \\ Marina Gazzano Baladi*** \\ Raul Renato Cardozo de Mello Tucunduva Neto
}

\begin{abstract}
RESUMO
As fáscias cervicais consistem em três lâminas: superficial, pré-traqueal e pré-vertebral. Elas sustentam vísceras, músculos, vasos e linfonodos profundos. Além disso, formam planos de clivagem naturais pelos quais os tecidos podem ser separados durante um procedimento cirúrgico e limitam a disseminação de processos infecciosos, como abscessos e/ou celulite profunda ${ }^{1}$. Um padrão de celulite é a angina de Ludwig (AL), que se desenvolve como extensão de uma infecção aguda de um molar inferior em cerca de $70 \%$ dos $\operatorname{casos}^{2}$. Assim que a infecção penetra no espaço submandibular, pode se estender para o espaço faríngeo lateral e, posteriormente, ao espaço retrofaríngeo, podendo resultar em extensões graves. Pode causar mediastinite descendente necrosante (MDN), que representa uma forma grave e rara de infecção, que exige diagnóstico precoce e tratamento cirúrgico para reduzir a mortalidade associada à doença ${ }^{3}$. O diagnóstico é baseado em exames clínicos e imaginológicos buscando determinar a severidade da infecção ${ }^{4}$. A ultrassonografia pode demonstrar a presença de coleções e infiltrações locais precocemente, o que facilita o diagnóstico. Os exames por tomografia computadorizada indicam a extensão da lesão, o comprometimento das vias aéreas e a presença de gases entremeados nos tecidos musculares. Pode-se avaliar o grau de comprometimento da região torácica quando afetada.
\end{abstract}

DESCRITORES: Angina de Ludwig • Ultrassonografia • Tomografia computadorizada

\section{ABS TRACT}

The cervical fascia consists of three layers: superficial, pretracheal and prevertebral. They contend viscera, muscles, blood vessels and lymph nodes. Furthermore they form natural cleavage planes through which tissues can be separated during a cirurgical procedure, and limit the spread of infectious processes such as abscesses and/or cellulite 1 . A pattern of cellulite is Ludwig's angina which develops as an extension of an accurate infection of a molar tooth in $70 \%$ of cases $^{2}$. Once the infection enters the submandibular space, may extend into the lateral pharyngeal space and subsequently to the retropharyngeal space, which can result in serious extensions. It may cause descending necrotizing mediastinitis which is a rare and severe form of infection that requires early diagnosis and surgical treatment to reduce mortality associated with the disease ${ }^{3}$. Diagnosis is based on clinical and imaging seeking to determine the severity of the infection ${ }^{4}$. Ultrasonography can demonstrate the presence of local infiltration and early collections, which facilitates the diagnosis. The examinations using computed tomography indicate the extent of the injury, the airway involvement and the presence of gas interspersed in muscle tissues. One can assess the degree of impairment of the thoracic region when affected.

DESCRIPTORS: Ludwig's angina • Ultrasonography • Computed tomography

* Prof Dr da Universidade Cidade de São Paulo

** Acadêmica do Curso de Medicina da Universidade Cidade de São Paulo

*** Pós graduanda da Universidade de São Paulo

**** Médico responsável pela Clínica Tucunduva 


\section{N T RO DUÇ Ã O}

As fáscias cervicais consistem em três lâminas de tecido conjuntivo: superficial, pré-traqueal e pré-vertebral. Elas sustentam vísceras, músculos, vasos e linfonodos profundos e formam planos de clivagem naturais pelos quais os tecidos podem ser separados durante a cirurgia e limitam a disseminação de abcessos. Tais membranas permitem que as estruturas do pescoço movimentem-se sem dificuldades ${ }^{1,5}$.

Quando um abscesso não é capaz de drenar através da superfície cutânea ou para o interior da cavidade oral, ele pode estender-se difusamente pelos planos fasciais que envolvem outras estruturas de partes moles. Essa extensão aguda e edemaciada de um processo inflamatório agudo é denominada de celulite ${ }^{6}$. Os abscessos podem também resultar de bactérias no assoalho da boca, na base da língua, nas tonsilas ou a partir de injeções intravenosas (IV) administradas na veia jugular interna (especialmente entre abusadores de drogas). Problemas de desidratação secundária e a incapacidade de engolir podem levar à osteomielite e às infecções mediastinais ${ }^{4}$.

Um padrão de celulite é a angina de Ludwig ( $\mathrm{AL}$ ), que se desenvolve da extensão de uma infecção aguda de um molar inferior em cerca de $70 \%$ dos casos. Assim que a infecção penetra no espaço submandibular, pode se estender para o espaço faríngeo lateral e, posteriormente, ao retrofaríngeo, podendo resultar em extensões graves. Além de afetar as estruturas periorbitais, a infecção pode estender-se para o seio cavernoso, na base do crânio, e causar trombose desse seio ${ }^{6}$.

A angina é utilizada para descrever a ameaça de sufocamento observada nesses pacientes. Alguns autores a chamam de angina maligna ou estrangulamento mórbido. A AL é um quadro clássico, descrito em 1836 por Wilhelm Von Ludwig, sendo uma celulite frequentemente originada de uma infecção odontogênica classicamente localizada no segundo e terceiro molares inferiores, que envolve os espaços submandibular, sublingual e submentual ${ }^{2}$.

A AL caracteriza-se por uma tumefação no assoalho da boca, língua e região submandibular. Inicialmente unilateral, porém ocorre a extensão para a porção contralateral do pescoço ${ }^{6}$.

Quando ocorre o envolvimento bilateral de mais de um espaço cervical, ocorre gangrena com infiltração serosa sanguinolenta putrefata com pouco ou nenhum pus externo apreciado, o envolvimento do tecido conjuntivo, a fáscia, músculos, mas o tecido não glandular. O processo infeccioso é muitas vezes transmitido através do espaço fascial, mas não pelo sistema linfático ${ }^{7}$.

Pode ser observada dor no pescoço e no assoalho da boca, restrição dos movimentos do pescoço, disfalgia, disfonia, disartria, sialorreia, garganta dolorida, febre, edema do pescoço e ao redor dos tecidos moles, trismo, edema da língua, edema da glote, protrusão lingual, linfadenopatia e calafrios. Taquipneia, dispneia, taquicardia, sibilo, inquietação e a necessidade do paciente de manter uma postura ereta sugerem a obstrução das vias aéreas, pelo edema da laringe. Sintomas adicionais podem envolver os espaços sublinguais e pode-se observar a lateralização da orofaringe e do osso hioide devido à elevada concentração de coleção purulenta ${ }^{2,4,6}$.

Fatores que contribuem para $\mathrm{AL}$ são infecção odontogênica, fratura de mandíbula, presença de corpo estranho no assoalho bucal, abscesso peritonsilar e faríngeo, linfangiomas, otite média e outros danos para o interior da cavidade oral. Inicia-se por uma infecção dental, com subsequente perfuração da cortical mandíbular lingual. o que leva a uma progressão do processo nos espaços submandibular (SMS), sublingual (SLS) e submentual (ESMe). Sendo mais complicado em pacientes que apresentam glomerulonefrite crônica, lupus sistêmico eritematoso, imunodeficiência e diabetes mellitus. As crianças com sistema de defesa deficiente são mais susceptíveis à contaminação por microrganismos oportunistas ${ }^{2,7,8}$.

Pode causar mediastinite descendente necrosante (MDN), como consequência, que representa uma forma grave e rara de infecção mediastinal, a qual exige diagnóstico precoce e tratamento cirúrgico para reduzir a alta mortalidade associada a essa doença. A presença de dor abdomi-
TUCUNDUVA MJAPS FREITAS CF OGASSAVARA B BALADI MG

TUCUNDUVA neto $R R C M$

ESTUDO

IMAGINOLÓGICO

DE UM CASO

DE ANGINA DE

LUDWIG

165
REV, ODONTOL.

UNIV. CID, SÃo

PAULO

2013; 25 (2):

$164-9$, MAIO-AGO 
TUCUNDUVA MJAPS:

FREITAS CF

OGASSAVARA B

BALADI MG

TUCUNDUVA NETO

RRCM

ESTUDO

IMAG INOLÓG I CO

DE UM CASO

DE ANGINA DE

LUDWIG

166

REV, ODONTOL.

UNIV, CID, SÃO

PAULO

$2013 ; 25(2): 164-$

9, MAIO-AGO
I SSN 1983-5183

nal em paciente com MDN deve ser seriamente considerada, porque pode ser uma indicação de extensão retroperitoneal ${ }^{9}$.

Esses pacientes com MDN estão mais propensos a terem outras complicações como broncoaspiração, paralisia dos pares cranianos, abscessos cerebrais, fasciite necrosante, síndrome da angústia respiratória do adulto e falência de múltiplos órgãos. A ocorrência de erosão dos vasos cervicais com sangramento abundante não é uma complicação infrequente de infecções profundas do pescoço ${ }^{9}$.

Para o diagnóstico de $A L$, as seguintes características devem ser observadas: inicia-se no assoalho da boca, geralmente com a infecção do $2^{\circ}$ ou $3^{\circ}$ molar inferior; a disseminação ocorre para o espaço submandibular, mais por contiguidade, pelos planos das fáscias, do que pelos vasos linfáticos; a infecção apresenta-se como endurecimento da região submandibular, sem formação de muita secreção purulenta; o processo poupa as glândulas salivares e linfonodos; é geralmente bilateral ${ }^{9}$.

O diagnóstico de infecção cervical é clinicamente óbvio, mas o diagnóstico precoce de mediastinite é mais difícil, devendo-se suspeitar quando há sintomas respiratórios como: dor torácica, dispneia ou angústia respiratória associada a abscesso cervical ${ }^{9}$.

O diagnóstico baseado em exames clínicos e imaginológicos aliados a medicação efetiva e intervenção cirúrgica precoce são primordiais para a manutenção da vida do paciente. O retardo do diagnóstico implica em maior disseminação da infecção².

Os métodos de imagem podem determinar a severidade da infecção. A ultrassonografia pode demonstrar a presença de coleções purulentas. Radiografias planas convencionais são úteis na abordagem inicial, para se observar o possível foco dentário infeccioso. As radiografias do pescoço e do tórax podem revelar várias características que aparecem tardiamente no curso da doença; contudo, procedimentos prévios (drenagem cervical, traqueostomia e acesso venoso central) tornam difícil a interpretação das radiografias de tórax ${ }^{9}$.

Os exames por tomografia computado- rizada e ressonância magnética indicam a extensão da lesão, o comprometimento das vias aéreas e a presença de gases entremeados nos tecidos musculares. Pode-se avaliar, também, o comprometimento da região torácica, quando afetada, permitindo assim a localização ideal à intervenção cirúrgica².

A AL é uma emergência cirúrgica rara que é potencialmente fatal, a menos que cedo reconhecida e tratada agressivamente. O tratamento adequado seria eliminação do foco infeccioso original, a manutenção das vias aéreas, antibioticoterapia e, por último, drenagem cirúrgica, se houver a presença de pus, a qual é rara. Se os sinais e sintomas de uma iminente obstrução das vias aéreas se desenvolverem, deverá ser executada a intubação endotraqueal ou a traqueostomia ${ }^{2,6,8}$.

Quando a infecção apresenta ponto de flutuação, a intervenção cirúrgica é indicada, para desbridamento e drenagem dos espaços infeccionados. Utiliza-se drenagem na região por alguns dias para facilitar o escoamento da coleção purulenta. Agentes antimicrobianos apresentam um importante papel no tratamento dessas infecções. A escolha do medicamento deve ser de acordo com o tipo de bactéria, via de administração, baixo nível de toxicidade e custo razoável. Na maioria dos casos, a infecção é polimicrobiana, causada principalmente por estreptococos e estafilococos. A penicilina é o antibiótico de escolha, recomendado para o tratamento de infecções orofaciais. Pode ser substituído por clindamicina em pacientes alérgi$\cos ^{2}$.

A drenagem mediastinal transcervical está justificada em pacientes com doença limitada ao mediastino superior. Porém, sepse com comprometimento extenso do mediastino requer drenagem através de toracotomia sem demora, resultando em um quadro clínico grave descrito como mediastinite descendente necrosante $(\mathrm{MDN})^{9}$.

Antes dos modernos antibióticos, a mortalidade pela $\mathrm{AL}$ frequentemente excedia os $50 \%$. Mesmo que essa taxa tenha sido reduzida a menos de $10 \%$, ainda ocorrem mortes por suas complicações, como pneumonia, mediastinite, septice- 
mia, empiema e obstrução respiratória ${ }^{2,10}$.

Pacientes com MDN que tiveram drenagem mediastinal pelo acesso de Clamshell, o qual inclui toracotomia anterior bilateral com esternotomia transversa, mostraram vantagens com esse acesso que são: cirurgia em um único tempo, excelente exposição para descorticação bilateral, desbridamento do mediastino e pericardiectomia. Porém, essa cirurgia é particularmente invasiva nos pacientes de casos extremamente graves, expondo-os ao risco de paralisia do nervo frênico, osteomielite do esterno, deiscência de sutura do esterno e risco de óbito mais elevado $^{9,11}$.

A esternotomia mediana também parece inadequada em $M D N$, pela incidência de osteomielite, deiscência de sutura do esterno e por ser o acesso para o

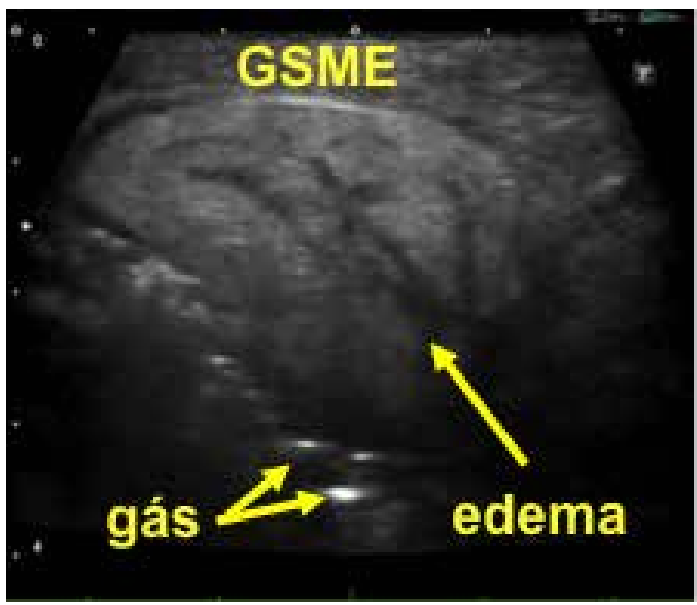

Figura 1 - Imagem de USG, no eixo longitudinal da glândula submandibular esquerda, com infiltrado inflamatório com líquido (hipoecóico) e gás (hiperecóico).

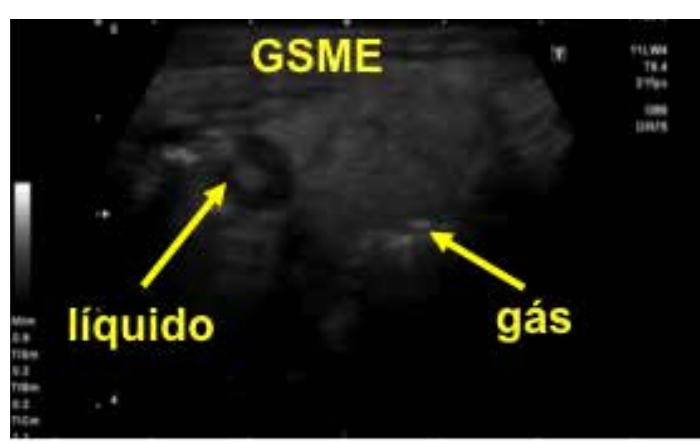

Figura 3 - Imagem de USG, no eixo transverso da glândula submandibular esquerda apresentando-se edemaciada. compartimento póstero-basal da cavidade torácica inadequado, especialmente no lado esquerdo9.

\section{RELATO DE CASO}

Paciente do gênero masculino, leucoderma, 27 anos de idade cronológica, sofreu manipulação dos canais do elemento de 24 horas antes. No exame físico apresentou febre, muito edema e dor local. Foi realizada a ultrassonografia da região supra-hióidea e cervical baixa, sendo para essa exploração utilizado o transdutor de 3,5 $\mathrm{MHz}$ (Figuras 1 a 4).

Foi observado edema da pele e do tecido celular subcutâneo, em hemiface esquerda, na região submandibular. As estruturas vasculares mostraram-se de configuração anatômica normal, os planos musculares e adiposos conservados.

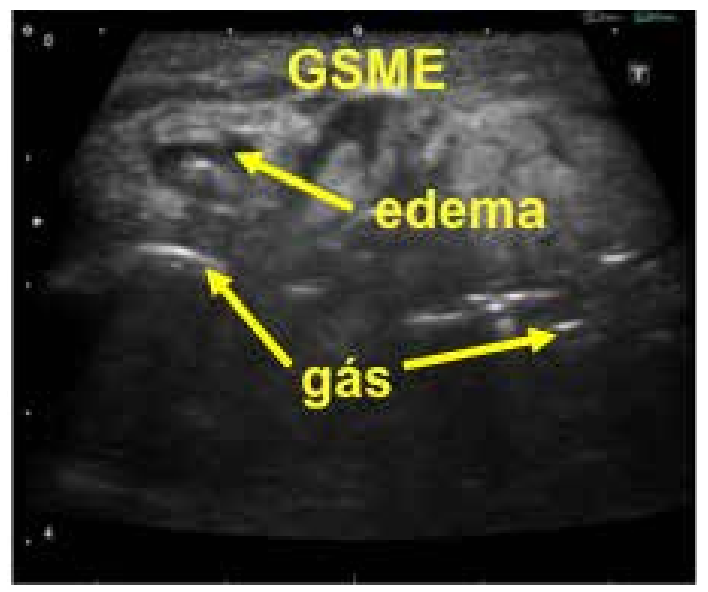

Figura 2 - Imagem de USG, no eixo longitudinal da glândula submandibular esquerda, com infiltrado inflamatório com líquido (hipoecóico) e gás (hiperecóico).

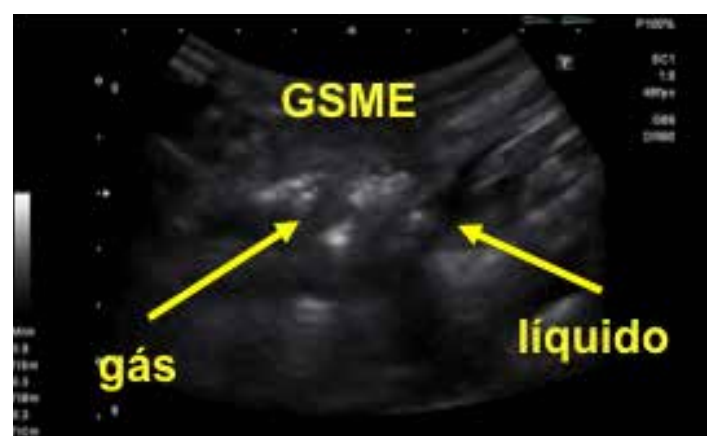

Figura 4 - Imagem de USG, no eixo transverso da glândula submandibular esquerda, com infiltrado inflamatório com líquido (hipoecóico) e gás (hiperecóico).
TUCUNDUVA MJAPS FREITAS CF

OGASSAVARA B

BALADI MG

TUCUNDUVA NETO $R R C M$

ESTUDO

IMAGINOLÓGICO

DE UM CASO

DE ANGINA DE

LUDWIG

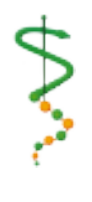

REV, ODONTOL. UNIV, CID, SÃO PAULO

2013; 25(2): 164-9, MAIO-AGO 
TUCUNDUVA MJAPS

FREITAS CF

OGASSAVARA B

$B A L A D I \quad M G$

TUCUNDUVA NETO

RRCM

ESTUDO

IMAGINOLÓG ICO

DE UM CASO

DE ANGINA DE

$\angle U D W I G$

168

REV, ODONTOL,

UNIV, CID, SÃO

PAULO

2013; 25(2): 164 -

9, MAIO-AGO
ISSN 1983-5183

Houve evidência de linfonodomegalias locais, com acentuado aumento da glândula submandibular esquerda, ao redor da qual se observam artefatos compatíveis com a presença de gás e líquido, sugestivos de infecção anaeróbia. Notou-se possível coleção em parede lateroposterior esquerda da faringe, o que foi indicativo de estar a infecção disseminando-se para os planos profundos e posteriores. Em se- guida, procedeu-se ao exame de tomografia computadorizada (figuras 5 a 7 - tomografia computadorizada do crânio, figuras 8 e 9 - tomografia computadorizada da região cervical), que confirmou os achados ultrassonográficos, ficando estabelecido o diagnóstico de angina de Ludwig. Foi feita a drenagem do abscesso e houve restabelecimento do quadro de normalidade do paciente.

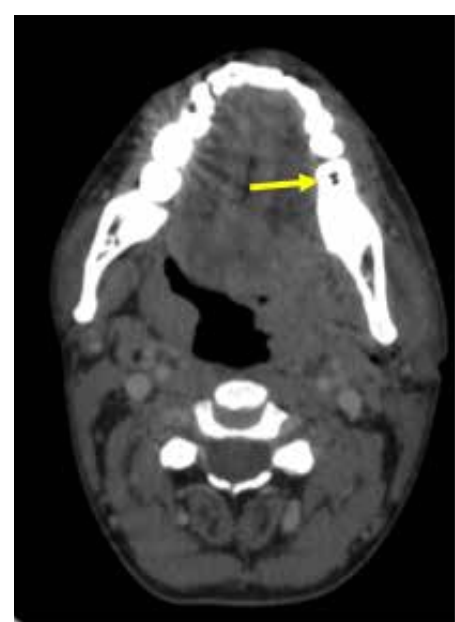

Figura 5 - TC axial da mandíbula mostrando o elemento 46 com abertura endodôntica.

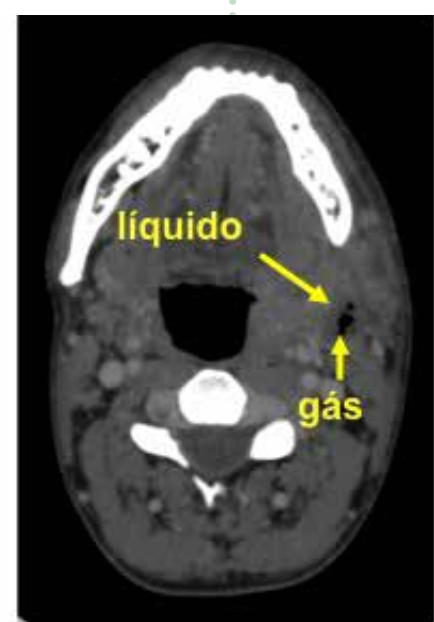

Figura 6 - TC axial da região cervical alta com infiltrado inflamatório à esquerda: gás (hipodenso) e líquido (isodenso).

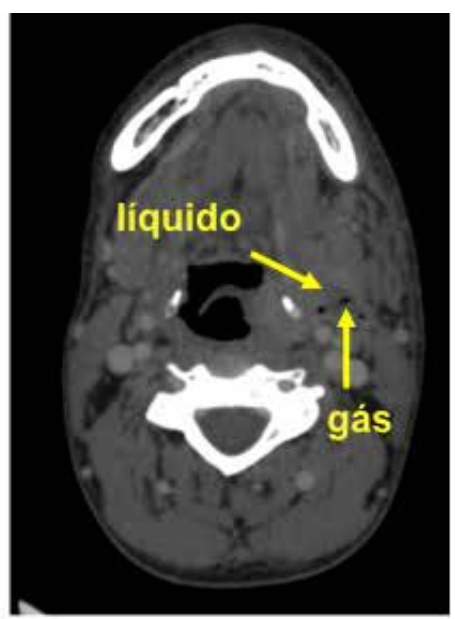

Figura $7-T C$ axial da região cervical alta com infiltrado inflamatório à esquerda: gás (hipodenso) e líquido (isodenso).

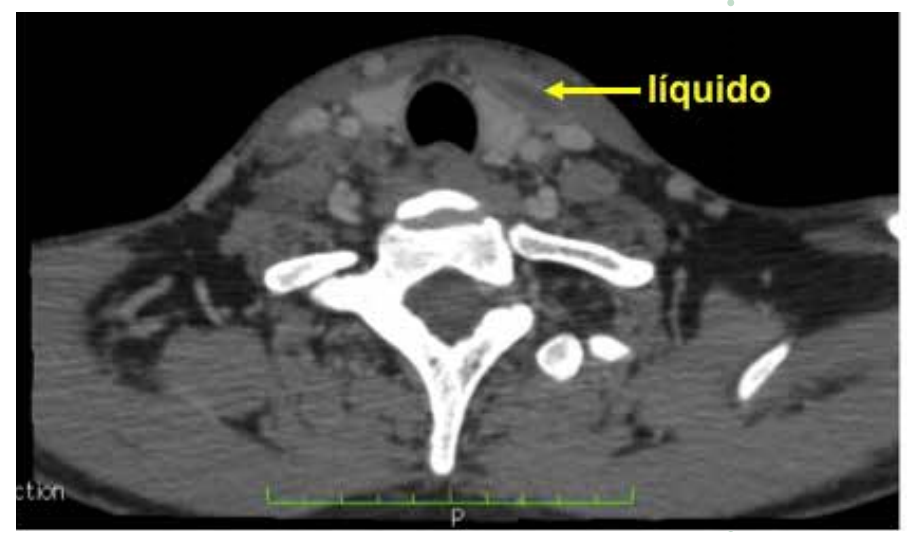

Figura 8- TC axial da região cervical baixa mostrando coleção dissecando os planos superficiais à esquerda.

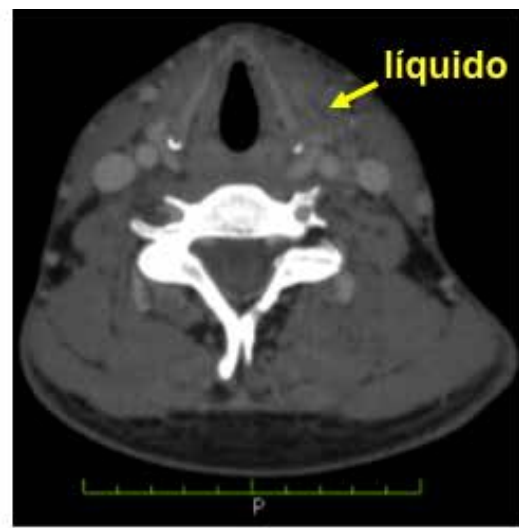

Figura 9 - TC axial da região cervical baixa mostrando coleção dissecando os planos superficiais à esquerda. 
TUCUNDUVA MJAPS

FREITAS CF

OGASSAVARA B

BALADI MG

TUCUNDUVA NETO $R R C M$

ESTUDO

IMAGINOLÓGICO

DE UM CASO

DE ANGINA DE

LUDWIG
3. Neff SP, Merry AF, Anderson B. Airway management in Ludwig's angina. Anaesth Intensive Care 1999 Dec;27(6):659-61.

4. Honrado CP, Lam SM, Karen M. Bilateral submandibular gland infection presenting as Ludwig's angina: first report of a case. Ear Nose Throat J 2001 Apr;80(4):217-8, 22-3.

5. Hamilton GF, Fyfe FW, Lockhart RD, editors. Anatomia do corpo humano. 2. ed. Rio de Janeiro: Guanabara Koogan; 1986.

6. Neville BW. Patologia oral \& maxilofacial. 2. ed. Rio de Janeiro Guanabara Koogan; 2004. ditions with airway implications. 2012 [Acesso em março de 2012]; Disponível em: http://www.embbs.com/aem/ neckd.html.

8. Hasan W, Leonard D, Russell J. Ludwig's Angina-A Controversial Surgical Emergency: How We Do It. Int J Otolaryngol $20112011(231816$.

9. Brommelstroet $M$, Rosa JFT, Boscar$\operatorname{dim}$ PCB, Schmidlin CA, Shibata S. Mediastinite descendente necrosante pós-angina de Ludwig. J Pneumologia 2001 Sept.;27(5):269-71.

10. Busch RF. Ludwig angina: early aggressive therapy. Arch Surg Otolaryngol Head Neck 1999 125(11):1283-4.

11. Hartmann RW, Jr. Ludwig's angina in children. Am Fam Physician 1999 Jul;60(1):109-12.

Recebido em 18-04-2013

Aceito em 19-06-2013

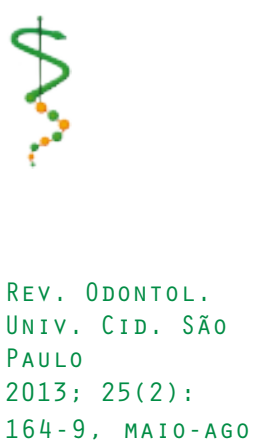

